\title{
Scaling up phenotypic plasticity with hierarchical population models
}

\author{
Eelke Jongejans $・$ Heidrun Huber $\cdot$ Hans de Kroon
}

Received: 24 March 2009/Accepted: 27 November 2009/Published online: 12 January 2010

(C) Springer Science+Business Media B.V. 2010

\begin{abstract}
Individuals respond to different environments by developing different phenotypes, which is generally seen as a mechanism through which individuals can buffer adverse environmental conditions and increase their fitness. To understand the consequences of phenotypic plasticity it is necessary to study how changing a particular trait of an individual affects either its survival, growth, reproduction or a combination of these demographic vital rates (i.e. fitness components). Integrating vital rate changes due to phenotypic plasticity into models of population dynamics allows detailed study of how phenotypic changes scale up to higher levels of integration and forms an excellent tool to distinguish those plastic trait changes that really matter at the population level. A modeling approach also facilitates studying systems that are even more complex: traits and vital rates often co-vary or trade-off with other traits that may show plastic responses over environmental gradients. Here we review recent developments in the literature on population models that attempt to include phenotypic plasticity with a range of evolutionary assumptions and modeling techniques. We present in detail a model framework in which environmental impacts on population dynamics can be followed analytically through direct and indirect pathways that importantly incorporate phenotypic plasticity, trait-trait and trait-vital rate relationships. We illustrate this framework with two case studies: the population-level consequences of phenotypic responses to nutrient enrichment of plant species occurring in nutrient-poor habitats and of responses to changes in flooding regimes due to climate change. We conclude with exciting prospects for further development of this framework: selection analyses, modeling advances and the inclusion of spatial dynamics by considering dispersal traits as well.
\end{abstract}

Electronic supplementary material The online version of this article (doi:10.1007/s10682-009-9340-2) contains supplementary material, which is available to authorized users.

E. Jongejans $(\bowtie) \cdot H$. Huber $\cdot$ H. de Kroon

Department of Experimental Plant Ecology, Radboud University Nijmegen, Heyendaalseweg 135, 6525 AJ Nijmegen, The Netherlands

e-mail: E.Jongejans@science.ru.nl

E. Jongejans

Nature Conservation and Plant Ecology Group, Wageningen University, Droevendaalsesteeg 3a, 6708

PB Wageningen, The Netherlands 
Keywords Life history components · Life table response experiments ·

Matrix projection models · Trait-trait covariances · Vital rates

\section{Introduction}

Plants can adapt to variable environments by changing their phenotype which typically is expected to increase individual fitness (Pigliucci 2001; Sultan and Stearns 2005; Bradshaw 2006). Despite the expectation that phenotypic plasticity (i.e. environmentally induced trait variation) will have important consequences for population dynamics at the local and landscape scale (Sultan 2007), studies of phenotypic plasticity typically focus on individual fitness. The effect of phenotypic plasticity across environments on fitness components like reproduction or survival can be analyzed statistically with path models or structural equation models. Path models fit hypothesized networks of causal relationships between ecological drivers, individual traits and one or more fitness components to data (Huber et al. 2004; Pigliucci and Kolodynska 2006; Picotte et al. 2007; de Vere et al. 2009). However, finding effects of phenotypic plasticity on a fitness component does not automatically allow for conclusions at the population level (Metcalf and Pavard 2007). The relationship between phenotypic plasticity and population dynamics is unlikely to be straightforward: phenotypic shifts in one trait may have indirect fitness consequences through positively or negatively (e.g. trade-offs) correlated traits (Tonsor and Scheiner 2007). Furthermore, changes in individual fitness rarely translate linearly into population size fluctuations (Ehrlén 2003), partly because not all fitness components are equally important for local population growth and partly because not all individuals will respond in the same way.

Evaluation of the population-level consequences of phenotypic plasticity requires computer simulations or, more elegantly, analytical population models (Caswell 1983). Matrix population models have proven to be very useful because they transparently represent the life cycle of a species by including all the year-to-year transitions between the various age or size stages in which individuals can be classified (Caswell 2001). These annual transitions are made up of vital rates (i.e. fitness components) such as stage-specific survival and reproduction rates and growth rates of surviving individuals that reach other stages. The mathematical characteristics of matrices have clear biological interpretations such as the projected population growth rate (i.e. the dominant eigenvalue of the transition matrix) and the relative contributions of matrix elements or vital rates to population growth (i.e. elasticity values; de Kroon et al. 2000; Franco and Silvertown 2004). Matrix models have continued to develop rapidly and now include stochasticity (Tuljapurkar et al. 2003) and a spatial dimension (Neubert and Caswell 2000), while still retaining all useful analytical properties.

Matrix population models have already been used to investigate the consequences of the outcome of phenotypic plasticity, for instance reduced variability in demographic rates due to dampening of the impact of environmental fluctuations (Caswell 1983). Temporal variation in demography is generally thought to decrease population growth (Tuljapurkar 1990; Boyce et al. 2006), although that still depends on the specific response (e.g. linear or convex) of a vital rate to an environmental driver (Koons et al. 2009). It has therefore been hypothesized that natural selection has led to the reduction of the variation of especially those vital rates that contribute most to the population growth rate (Pfister 1998; Morris and Doak 2004). However, these studies did not specifically include the plastic traits that may underlie vital rate variability. 
In this paper, we develop a framework of hierarchical population models (HPMs) to analyze the effects of phenotypic plasticity on demographic and dispersal traits at the population level. In this context we will investigate plastic changes of morphology, biomass accumulation, flowering probability and reproductive effort; traits are directly and indirectly linked with demography and dispersal processes. In essence, HPMs bring together two research lines: that of studying the effects of phenotypic plasticity with path models and that of spatial and non-spatial population modeling. This approach of coupling relationships between individual traits and vital rates inside matrix models was already pioneered by van Tienderen (2000) with an hypothetical plant species, and applied to and extended for animal field data by Coulson et al. (2003; 2006: Pelletier et al. 2007; Coulson and Tuljapurkar 2008). Here we develop HPMs for perennial plants and add spatial dynamics to the equation. We will illustrate how HPMs can be used to answer the following important questions: what are the population-level consequences of trait-trait covariance and how does phenotypic plasticity change the effect of environmental fluctuations on local and spatial population dynamics.

\section{Hierarchical population models}

Hierarchical population model (HPMs) can be schematically represented (as for instance in Fig. 1) in the same way as path models: environmental factors (i.e. ecological drivers such as flooding, nutrient availability, weather, or population density) influence traits of individuals, which in turn affect vital rates (or fitness components such as survival, growth and reproduction) that together can be used to build population models (e.g. a population transition matrix). Thus, each of these lower-level parameters (i.e. environmental factors, traits of individuals, vital rates) can influence population dynamics. Vice versa (from right to left in Fig. 1) the arrows leading to a model parameter indicate which lower-level parameters contribute to that higher-level parameter. Like in path models covariances between individual traits can be included in HPMs. Trade-offs among traits result in negative covariances. Depending on how individual traits of interest are defined, HPMs may also include direct effects of environmental factors on vital rates (' $v$ ' in Fig. 1). It is also possible that the changes in the environment affect how an individual trait contributes to a vital rate (i.e. the vital rate function of that trait changes with the environment).

However, to our knowledge no such complex hierarchical population models including all the relationships described above have been performed so far. In the next section we will present the results of a case study (Fig. 1) for which we have data and for which we show numerically (Fig. 2) what insights can be gained from a HPM approach. Thereafter we will explore a more complex, hypothetical case study which includes various environmental effects and trait-trait covariation (Fig. 3).

\section{Eutrophication effects on the population dynamics of 4 grassland species}

The first case study is an example of how an HPM can be constructed and analyzed. We analyzed the population-effect of eutrophication, which has caused declines in species richness in many grasslands (Neitzke 2001; Stevens et al. 2004). We focused on four perennial plant species (Centaurea jacea, Cirsium dissectum, Hypochaeris radicata and Succisa pratensis) of which the demography has been studied in nutrient-poor grasslands (Jongejans and de Kroon 2005; Jongejans et al. 2008). 


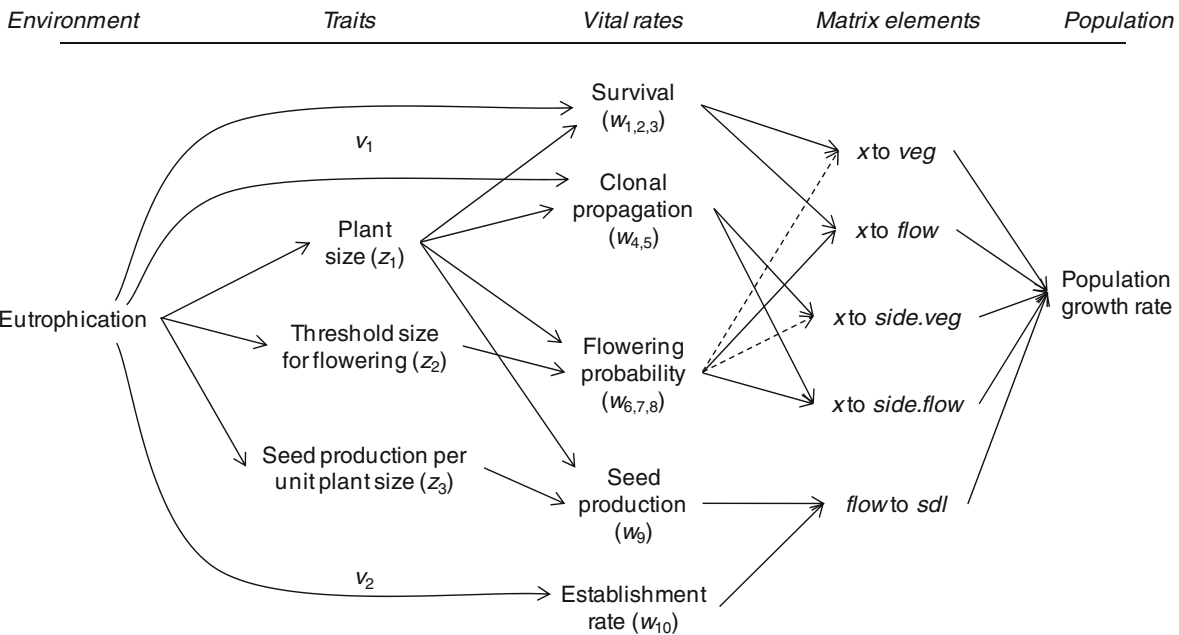

stage at time $t$

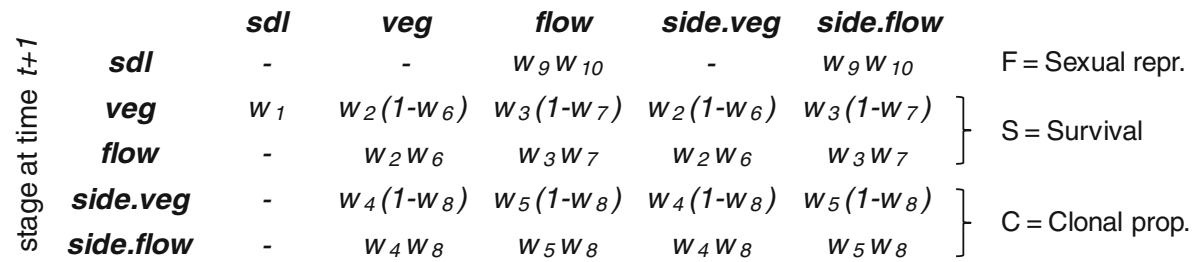

Fig. 1 (top) Hierarchical population model of the contributions of nutrient enrichment (environmental factor) to consecutively plant traits, vital rates, matrix elements and population growth (see Table 1 for definitions of all model parameters). Eutrophication affects plant traits $\left(z_{1}, z_{2}, z_{3}\right)$ but also directly $\left(v_{1}, v_{2}\right)$ affects the survival and establishment rates independent of changes in plant traits, for instance through changes in the surrounding vegetation. In this case we made the simplifying assumption that the investigated plant traits do not co-vary. (bottom) $5 \times 5$ 1-year transition matrix with 10 vital rates $\left(w_{k}\right)$. The top row contains sexual reproduction, the second and third rows contain survival elements, and the bottom two rows contain clonal propagation

To study the importance of lower-level parameters we formulated an HPM (see Fig. 1 for details) with the following plant traits: plant size $\left(z_{1}\right)$, threshold size for flowering $\left(z_{2}\right)$ and seed production per unit plant size $\left(z_{3}\right)$. With $z_{1}$ we fit linear models to the following vital rates: the number of clonal offspring per non-flowering $\left(w_{4}\right)$ and per flowering rosette $\left(w_{5}\right)$ and the number of seeds produced per flowering rosette $\left(w_{9}\right)$. The slope of the latter seed production model is the plant trait $z_{3}$, the number of seeds per unit plant size. For adult survival $\left(w_{2}, w_{3}\right)$ and flowering $\left(w_{6}, w_{7}, w_{8}\right)$ we performed generalized linear models with a logit-link and plant size as the explanatory variable. We inserted the average of observed plant sizes into these functions to obtain average vital rate values for the field scenario (see Supplementary Material for details).

The field scenario (i.e. control, nutrient-poor conditions) was contrasted with an eutrophication scenario, which was based on the field scenario, but altered at five points: the three plant traits $\left(z_{1}, z_{2}, z_{3}\right)$, and two direct effects $\left(v_{1}, v_{2}\right)$ on the vital rates survival and seedling establishment. For the changes in plant traits and survival we used the relative effects that were found in a garden experiment in which these four plant species were 
Table 1 Definition of all the stage classes and model parameters used in Fig. 1

\section{Param. Definition}

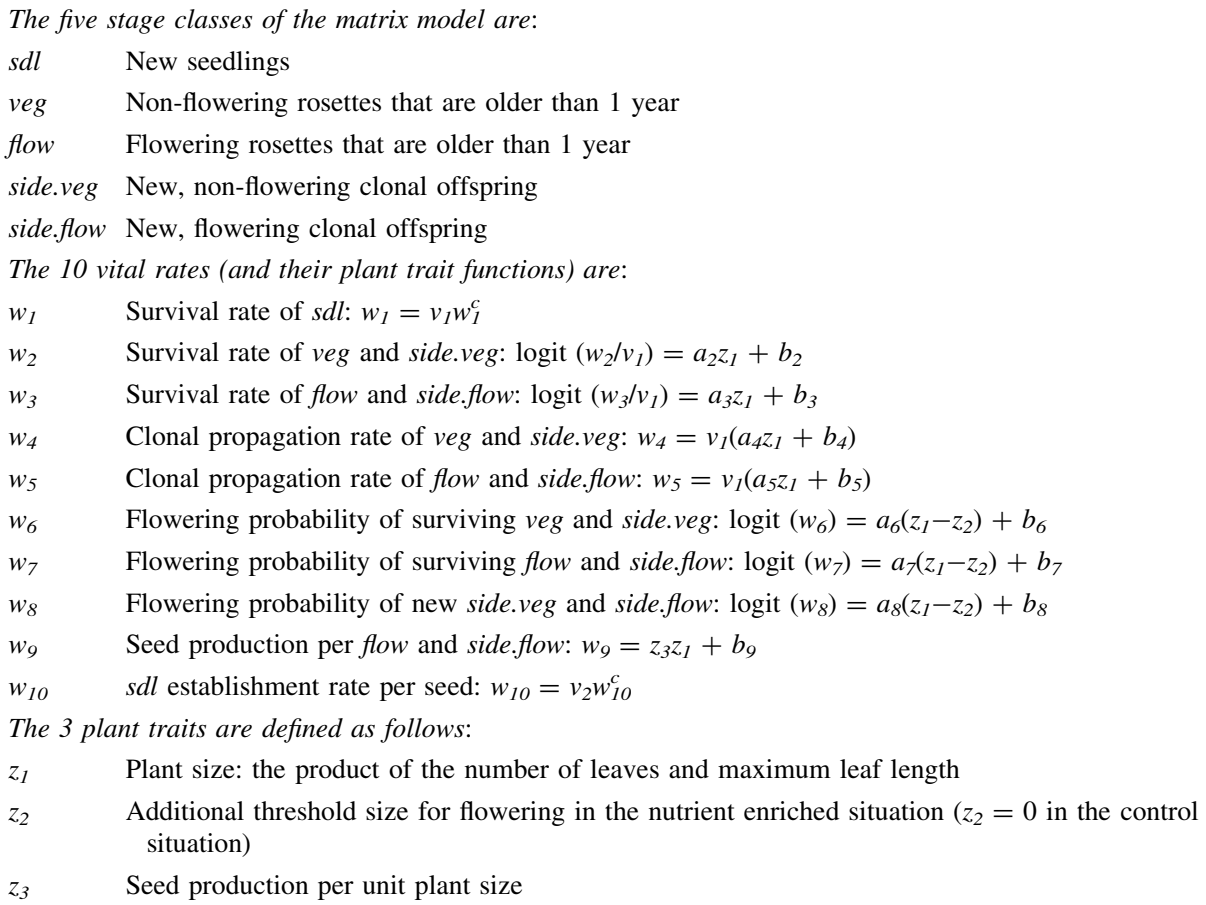

The 2 direct environmental effects on vital rates are:

$v_{1} \quad$ Effect of eutrophication on plant survival and clonal propagation (which also involves survival till the next year)

$v_{2} \quad$ Effect of eutrophication on seedling establishment

The C-index indicates the control, field scenario. The $a$ 's and $b$ 's are empirically determined constants (see Supplementary Material)

grown amidst a hexagon of tussocks of the competitive grass Molinia caerulea (Jongejans et al. 2006). Half of the plots in the garden were annually fertilized, and by comparing survival and the sizes of the survivors between the enriched and control plots we were able to estimate how much nutrient enrichment, as applied in the garden experiment, affects the mentioned plant traits and the adult survival rate. For the calculation of the vital rates of the eutrophication scenario we changed the mean plant traits of the field scenario proportionally to the experimental fertilization effect sizes which can be found in Table 2 (see Supplementary Material for details). For the relative effect of eutrophication on seedling establishment $\left(w_{10}\right)$ we used the ratio of the establishment rate in high productive field sites and the establishment ratio in low productive field sites as found in a published seed addition experiment involving 20 sites (Soons et al. 2005).

Next we wanted to know how these different effects of eutrophication on plant traits and vital rates contributed to the difference $(\Delta \lambda)$ between the projected population growth rate of the eutrophication scenario $\left(\lambda^{E}\right)$ and that of the default field scenario $\left(\lambda^{C}\right)$. We therefore decomposed $\Delta \lambda$ with a so-called fixed-effect LTRE (i.e. Life Table Response Experiment; Horvitz et al. 1997; Caswell 2001; Jongejans and de Kroon 2005) to investigate at each 
LTRE analysis of a hierarchical population model

$\Delta \lambda$ Matrix elements Vital rates

Traits

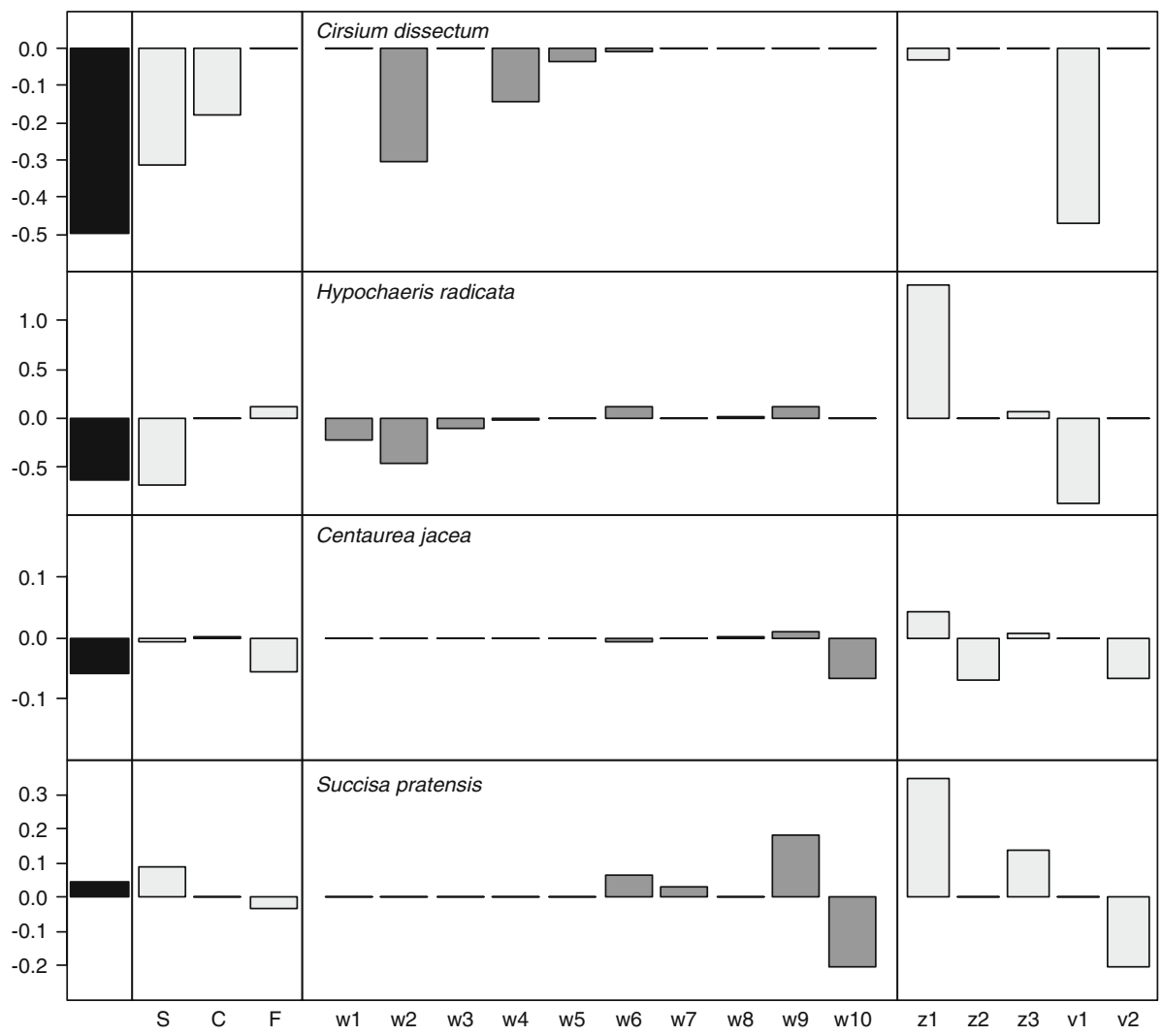

Fig. 2 For each of four grassland herb species (rows): Decomposition (LTRE) of the differences in projected population growth $(\Delta \lambda$, between the nutrient enriched scenario and the default field scenario) into contributions of differences in model parameters at three different levels: groups of matrix elements (S, C, $\mathrm{F})$, vital rates $\left(w_{k}\right)$, and plant traits $\left(z_{k}\right.$; see Table 1 for an explanation of the vital rates and plant traits). The matrix elements are grouped as in Fig. 1: $\mathrm{F}=$ sexual reproduction, $\mathrm{S}=$ survival, $\mathrm{C}=$ clonal propagation. At the plant trait level $\Delta \lambda$ is not only decomposed into contributions of the changes in the plants traits themselves $\left(z_{k}\right)$, but also into contributions of the effects of the environment $\left(v_{h}\right)$ on vital rates that do not involve plant traits. In each of the panels the LTRE contributions together approximate $\Delta \lambda$ given at the left

level what caused the difference between $\lambda^{C}$ and $\lambda^{E}$. LTREs approximate these contributions to $\Delta \lambda$ with the products of 1) the sensitivity of $\lambda$ to changes in a parameter and 2) the deviation of the value of that parameter from its control value (see Supplementary Material for the sensitivity and LTRE equations used for the trait, vital rate and matrix element levels). LTRE contributions of underlying parameters quantify the importance of those parameters for the given difference in $\lambda$ and together the contributions sum up to the total $\lambda$-difference observed. First we decomposed $\Delta \lambda$ at the level of the matrix elements $\left(a_{i j}\right)$, then at the level of the underlying vital rates $\left(w_{k}\right)$, and finally at the level of the involved plant traits $\left(z_{r}\right)$. The last level also included the contributions of changes in direct environmental effects $\left(v_{h}\right)$ on vital rates (see Fig. 1). This way the sum of the LTRE contributions at each level approximated $\Delta \lambda$. 
Table 2 The experimentally determined relative impacts of nutrient enrichment on different plant traits and vital rates

\begin{tabular}{lllllll}
\hline Affected plant traits and vital rates & $\begin{array}{l}\text { Control, field } \\
\text { scenario }\end{array}$ & \begin{tabular}{l} 
Eutrophication scenario \\
\cline { 5 - 6 }
\end{tabular} & $\begin{array}{l}\text { Cirsium } \\
\text { dissectum }\end{array}$ & $\begin{array}{l}\text { Hypochaeris } \\
\text { radicata }\end{array}$ & $\begin{array}{l}\text { Centaurea } \\
\text { jacea }\end{array}$ & $\begin{array}{l}\text { Succisa } \\
\text { pratensis }\end{array}$ \\
\hline $\begin{array}{l}\text { Plant size }\left(z_{1}\right) \\
\begin{array}{l}\text { Additional threshold size for } \\
\text { flowering }\left(z_{2}\right)\end{array}\end{array}$ & 1.00 & 0.81 & 1.68 & 1.44 & 1.34 \\
$\begin{array}{l}\text { Seed production per unit plant size } \\
\quad\left(z_{3}\right)\end{array}$ & 1.00 & 1.00 & 1.93 & 2.02 & 2.48 \\
$\begin{array}{c}\text { Direct effect on plant survival and } \\
\text { clonal propagation }\left(v_{1}\right)\end{array}$ & 1.00 & 0.53 & 0.15 & 1.00 & 1.00 \\
$\begin{array}{c}\text { Direct effect on seedling } \\
\text { establishment }\left(v_{2}\right)\end{array}$ & 1.00 & 0.00 & 1.00 & 0.00 & 0.00 \\
\hline
\end{tabular}

The control, field scenario values apply to all four studied grassland plant species

Eutrophication had a larger impact on $\lambda$ of the two shorter-lived species: $\Delta \lambda$ was -0.626 (from $\lambda^{C}=0.960$ to $\lambda^{E}=0.334$ ) for Hypochaeris radicata and -0.496 (from 1.007 to 0.511 ) for Cirsium dissectum, while only -0.059 (from $\lambda^{C}=0.986$ to $\lambda^{E}=0.928$ ) for Centaurea jacea and even +0.045 (from $\lambda^{C}=1.237$ to $\lambda^{E}=1.282$ ) for Succisa pratensis. Furthermore, the LTREs clearly showed that nutrient enrichment affected the population dynamics of these four grassland species differently (Fig. 2): at the level of matrix elements we see that the steep decline in $\lambda$ in the eutrophication scenario for the short-lived species was mostly caused by decreased survival (and by decreased clonal propagation for $C$. dissectum). However, reduced sexual reproduction had the largest negative contributions to $\Delta \lambda$ in the two longer-lived species (C. jacea and S. pratensis), although this was more than compensated in $S$. pratensis by positive contributions of matrix elements that represented the fate of surviving individuals.

At a lower level we see that this negative contribution of sexual reproduction in the longlived species is mainly caused by reduced seedling establishment $\left(w_{10}\right)$. At this vital rate level it becomes clear that the largest buffering of the lower establishment rate in S. pratensis actually takes place within the sexual reproduction matrix elements by increased seed production $\left(w_{9}\right)$. The vital rate analysis also shows that it is not the survival rate itself that contributed to a higher $\lambda$ in the eutrophication scenario, but that this was caused by higher flowering probabilities of surviving plants $\left(w_{6}, w_{7}\right)$. For the short-lived species it was mainly the reduction in the survival rate of non-flowering plants $\left(w_{2}\right)$ that caused the $\lambda$-declines.

Direct effects $\left(v_{1}\right.$ and $\left.v_{2}\right)$ of eutrophication had the largest negative contributions at the lowest level (see right column of Fig. 2). These negative effects on $\lambda$ were to some extent buffered by positive contributions by changed plant traits, showing that plastic responses to eutrophication of plants that are growing amidst competitors can indeed be beneficial for population growth. Increased plant size and increased seed production per unit biomass were especially important in $S$. pratensis, and less so in $H$. radicata and $C$. jacea. The last species, $C$. dissectum, did not show any of these plastic responses and it might well be that that contributes to the vulnerability of this declining Red list species (see also Jongejans et al. 2008; de Vere et al. 2009). Together these multi-level LTRE analyses showed which traits show plastic responses that are actually important for population growth and how the 
importance of phenotypic plasticity relates to direct environmental impacts. These analyses also showed which vital rates, and subsequently matrix elements, were affected most.

\section{Phenotypic plasticity in response to flooding}

In the second case study we explore a more complex situation. In disturbed habitats such as river floodplains, traits like root aerenchyma, anaerobic metabolism and rapid petiole elongation are essential for survival during summer floods (van der Sman et al. 1993; Mommer et al. 2006). Other traits like seed buoyancy and traits that affect the amount and timing of seed production (van Splunder et al. 1995; Boedeltje et al. 2004) also play crucial roles in the dynamics of plant populations. Variation in these plant traits among species in experiments has been shown to adequately explain the altitudinal zonation of plant species in floodplains (van Eck et al. 2004; Voesenek et al. 2004; Lenssen and de Kroon 2005; van Eck et al. 2006). Environmentally induced trait variation enhances individual fitness in variable environments, and is thus expected to be particularly important for preventing large changes in population size in flood-prone areas (Verschoor et al. 2004; Miner et al. 2005). However, a trade-off between adaptations to survival during flooding and investments in reproduction (van der Sman et al. 1993) makes the effect of flood-related survival traits on population growth complex. It is therefore very insightful to formulate these relationships for this flooding system in a hierarchical population model (Fig. 3). With an HPM the importance at the population level of direct and indirect pathways through environment-trait, trait-trait, and environment/trait-vital rate relationships can be disentangled.

For instance the plastic formation of root aerenchyma in some plant species (or genotypes of the same species) in response to partial flooding enables oxygen to diffuse to the roots of these species, thereby increasing their survival (Visser et al. 2000; Huber et al. 2009). For our modeling exercise it is important to realize that not only the plant trait is changing with the environment (phenotypic plasticity; ' $p$ ' in Fig. 3), but also the dependency of the survival rate on the amount of aerenchyma in the roots (' $d$ ' in Fig. 3): aerenchyma is crucial for survival when submerged, but contributes little to survival under drained conditions. Additionally, flooding may also affect survival directly (' $v$ ' in Fig. 3), in a way that does not involve variation in any traits or through plant traits that are not included in the model.

Another plastic trait through which some plants can increase their survival when flooded is plant height, since elongated stems that reach the water surface can supply submerged plant parts with oxygen (Blom and Voesenek 1996; Pierik et al. 2009). On the other hand, constitutive plant height at which seeds are released during seed set is also a determinant in seed dispersal by wind. More research is needed to know if and how flooding-induced and constitutive plant height are related ('c' in Fig. 3; cf. Weijschedé et al. 2006). Since flooding itself can increase seed dispersal distances (' $v$ ' in Fig. 3; Blom and Voesenek 1996), it might be that flooding has both direct and indirect effects on dispersal. Water, wind and other dispersal vectors can be combined in so-called total dispersal kernels (Nathan 2007). Taking dispersal parameters into account may or may not matter for local population dynamics, but it is crucial when considering spatial population dynamics (Fig. 3).

Piecing all these relationships together might be challenging, but also very worthwhile. The HPM in Fig. 3 may for instance be applied to investigate how phenotypic plasticity allows some plant species to survive when flooding regimes change due to global climate 


\section{Hierarchical population model}

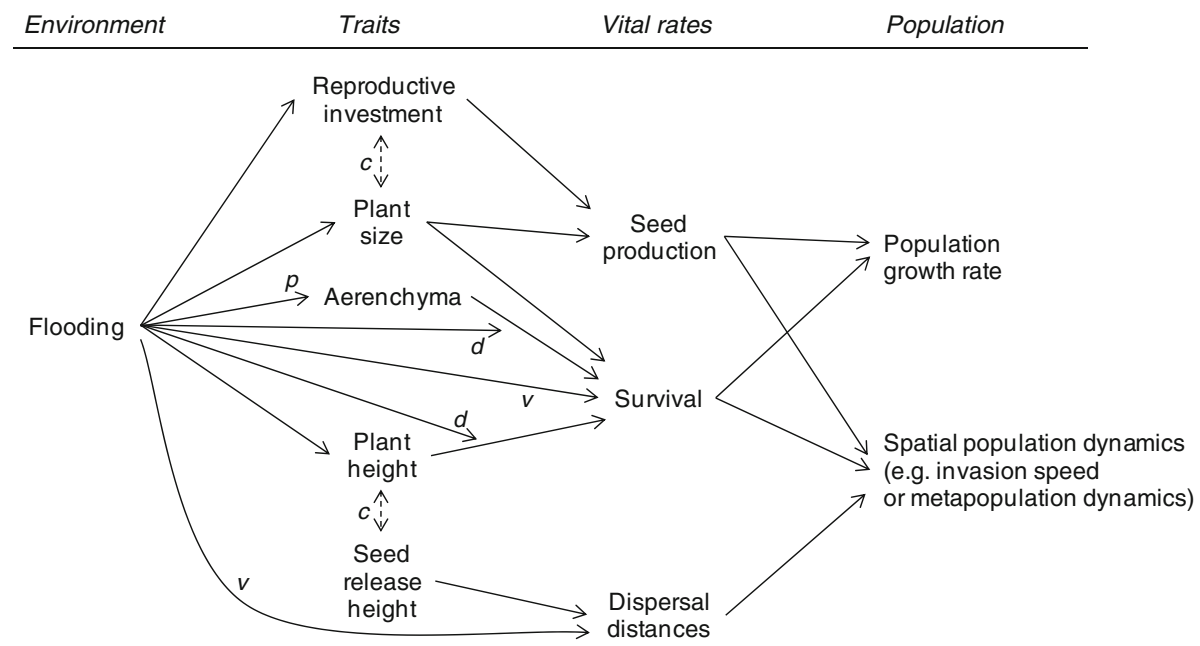

Fig. 3 An example of a hierarchical population model, in which environmental factors affect a population through a cascade of changes in traits of individuals and changes in vital rates. As illustrated by the arrows with letters, flooding may impact a population in three different ways: by $(v)$ directly affecting vital rates, by $(p)$ affected plant traits, or $(d)$ modifying relationships between vital rates and plant traits. The level of phenotypic plasticity determines how a trait responds $(p)$ to environmental changes (i.e. a reaction norm). The contribution of traits to vital rates can change $(d)$ with the environment: e.g. in this example the importance of plant height for survival depends on how much a plant is flooded. The environment can also affect vital rates directly $(v)$, in addition to effects through plant or seed traits; a flood can increase dispersal distances compared to an unflooded condition. Traits may co-vary $(c)$, for instance through allometry or trade-offs

change (e.g. shifted precipitation phenology and increased melting of glaciers). The approach outlined in Fig. 3 can, with relatively small adaptations of the input parameters and the underlying relationships, be used to study the response of populations to variation in other ecological drivers such as $\mathrm{CO}_{2}$, nutrient availability or salinity. Ultimately, HPMs can be used to test the promises of phenotypic plasticity as a mechanism for buffering effects of climate change, environmental stochasticity and habitat heterogeneity (Agrawal 2001; Callaway et al. 2003; Sultan 2007) by calculating the net effect of phenotypic plasticity at the population level.

Elderd and Doak (2006) compared the flooded and unflooded population dynamics of Mimulus guttatus, and found that of all the considered vital rates it was the increased germination rate and increased summer survival that caused the higher population growth in the flooded habitat. Similarly, Smith et al. (2005) found that population growth of the endangered Boltonia decurrens depends on the combination of early floods and precipitation. What HPMs could add to these interesting studies is to quantify how much plastic responses of the involved traits contributed to population growth in flooded habitats and to quantify the selection pressures on these traits in flooded and unflooded scenarios. Spatial HPMs are especially promising for comparing the roles of local plastic responses and escaping adverse conditions by dispersal. Such comparisons are especially of interest in the context of climate change and range shifts. A research agenda for population studies in floodplains could therefore include the following research questions: 
(a) How does phenotypic plasticity contribute to buffering environmental fluctuations at the local population level, and which plant traits and vital rates are directly and indirectly involved in mediating these fluctuations?

(b) To what extent can phenotypic plasticity buffer harsh environmental conditions in different landscape configurations, and how does it relate to the alternative strategy of escape by dispersal?

(c) Which life histories enable population persistence under past, present and future scenarios of flooding regimes, and is restoration management needed to prevent species loss as the climate continues to change?

Obviously, these research questions not just apply to flooding systems but also to any ecosystem where climate change is an important ecological driver and where the habitat ranges of species shift as a result of environmental changes.

\section{Discussion}

The pioneering hierarchical population model (HPM) of van Tienderen (2000) shows how traits like seed mass, germination time and flowering time shape the vital rates of a hypothetical annual plant. He quantified the direct effects of a trait on population growth via various vital rates and matrix elements as well as the indirect effects through correlations with other traits. Our first case study, in which we constructed a HPM with field and experimental data (Figs. 1,2), suggests that the potential of phenotypic plasticity to buffer detrimental environmental changes is species-dependent. In some species (e.g. S. pratensis) directly negative environmental impacts on survival seemed to have been buffered by increased plant size and sexual biomass allocation, whereas in other species (e.g. $C$. dissectum) such buffering by plastic responses was mostly absent. These exploratory studies suggest that HPM can indeed be very insightful for studying the importance of phenotypic plasticity for population dynamics under changing conditions.

\section{Studying phenotypic plasticity with HPMs}

In the eutrophication case study the incorporated traits were plant size and two traits that shape the amount of seed production (i.e. the threshold size for flowering and the number of seeds produced per unit plant size). In studies on phenotypic plasticity such biomassrelated traits are often considered to show 'passive' phenotypic plasticity because the change in plant size in response to nutrient enrichment may simply be a consequence of a direct relationship between resource availability and biomass accumulation which is not controlled by changes in developmental processes (Sultan 1995; van Kleunen and Fischer 2005; Kurashige and Callahan 2007). On the other hand, morphological changes such as stem elongation or aerenchyma formation in response to ecological drivers (Fig. 3) are seen as more 'active' phenotypic plasticity.

However, this distinction between active and passive phenotypic plasticity are not always easy to make. For instance, an increase in leaf size under shaded conditions can be considered an active foraging response (sensu Hutchings and de Kroon 1994) if it increases resource uptake compared to a non-plastic genotype. Shade induced reduction of leaf elongation, on the other hand, can be considered as reflecting a resource driven passive response (van Kleunen and Fischer 2005, 2007). A reduced leaf size under shaded conditions may also reflect an active response if it conserves resources and increases survival 
relative to a genotype producing larger leaves. The distinction between active and plastic responses can be of interest when comparing the genetic and physiological regulations of phenotypic plasticity at various stages of an individual's development. However, as shown in Figs. 1 and 3, different types of traits and responses (e.g. morphological, biomass, ratios) can be included in HPMs in similar ways. More important is the functionality of traits when deciding to incorporate them in HPMs: how well does a trait determine vital rates that are crucial parts of the life cycle, and thereby has the potential to significantly influence population dynamics.

Hierarchical population model (HPMs) are well-suited to study other important aspects of phenotypic plasticity as well. By comparing similar models that differ only in one or two key functions, the importance of including various degrees of phenotypic plasticity can be evaluated at the population level. The same methodology can be used to see if any costs of phenotypic plasticity, in terms of reduced growth and reproduction or costs through tradeoffs with other traits, are actually of significance. A wide variety of environmental conditions can be fed into stochastic Monte Carlo simulations to investigate to what extent phenotypic plasticity can mediate local and regional population persistence under variable conditions. Stochastic elasticities (Tuljapurkar et al. 2003) can then be used to analytically examine the simulation results and to see which model components contribute most to the population growth rates.

Another promising avenue in further developing HPMs is to incorporate continuous trait variables and plastic responses over the range of trait values, instead of merely studying changes in trait means. This could be achieved by adopting the methodology of integral projection models, which are similar to projection matrices but have continuous rather than discrete stage variables (Easterling et al. 2000; Rees and Rose 2002; Ellner and Rees 2006) and are therefore especially useful for studying the population consequences of environment-trait-life history relationships and their variances.

\section{Studying selection gradients with HPM}

van Tienderen (2000) calculated integrated elasticity values and selection gradients for individual traits while taking into account both the direct effects of a trait on population growth via various vital rates and matrix elements, and the indirect effects through correlations with other traits. One of the advantages of matrix population models for selection analysis is that an annual, integrated estimate of fitness can be distilled rather than a lifetime fitness approach, which does not take variation in life span among individuals into account (Coulson et al. 2006). Rees and Rose (2002) analyzed the selection pressure on the threshold size for flowering in a population of the monocarpic perennial Oenothera glazioviana, although they did not include any ecological driver or phenotypic plasticity. By changing input parameters (e.g. increasing or decreasing leaf size) one can test the effects of these changes not only on survival or seed output of plants, but also on growth of the whole population. If, for instance, reduction in biomass is associated to increased survival it may still ultimately result in positive (or less negative) population growth rates. Or it may not always be beneficial to increase seed set at the cost of survival if there are too few safe sites to ensure seedling establishment.

Hierarchical population models have successfully been developed and applied to detailed, long-term field data on red deer (Coulson et al. 2003) and soay sheep (Pelletier et al. 2007). The selection analyses in these studies are based on $\lambda$-sensitivity values (Coulson et al. 2003; sensitivity values also form the basis of the LTRE approach in Fig. 2), in contrast to a focus on proportional responses (i.e. elasticities, van Tienderen 
2000). These studies sophisticatedly show that traits like birth weight influence population growth through different vital rates, and that the contributions of these different pathways fluctuate strongly from year to year. Interestingly, these survival rates of different ages and genders responded differently to environmental drivers, resulting in no overall correlation between environmental fluctuation and selection (Coulson et al. 2003). Furthermore, the effect of positive selection on birth weight was buffered by the fact that birth weights of offspring tended to be smaller than those of the parents (Coulson and Tuljapurkar 2008).

Hierarchical population model (HPMs) are thus an excellent method to study trait selection and microevolution, because these models consider the role of a trait within the context of the entire life cycle and a set of ecological interactions (Metcalf and Pavard 2007; Knight et al. 2008). This would address one of the weaknesses of most studies on phenotypic plasticity where simply biomass or seed set is used as a fitness parameter to investigate which traits are under selection. Selection gradients may be very different for individual plants than for individuals that are part of a population and even more so for individuals in populations that are immersed in a community of different species. Ultimately, the seed production of a single plant needs to be evaluated with respect to the total number of seeds in the population in combination with the proportional germination and survival of seedlings to reproducing plants, in order to determine what the contribution of that plant is to future generations. HPMs might also prove useful tools for unraveling the population impact of trait variation further, at the genetic level (Metcalf and Mitchell-Olds 2009).

\section{Conclusions}

The future of hierarchical population models looks bright as new techniques are currently being developed to link statistically advanced path models to models of population dynamics. Statistically sound structural equation models also form a good tool for quantifying explained and unexplained variance at each higher-level upstream parameter (see e.g. Bakker et al. 2009 for a sophisticated analysis of the extinction risk of Californian island foxes). Information on explained variance is very useful for introducing individual and environmental stochasticity (Fox and Kendall 2002) into these local and spatial population models to study the importance of variation in individual traits due to environmental variation. As seen in this paper, HPMs bring together path analyses and population projection models, and form an excellent stage for studying the consequences of phenotypic plasticity for not only single fitness components, but importantly also for population dynamics.

Acknowledgments We are grateful to Eric Visser, Linda Jorritsma-Wienk, Pieter Zuidema, Juul Limpens, Gerard Bögemann and Annemiek Smit-Tiekstra for helpful discussions. Staatsbosbeheer kindly gave permission to perform the demography study in their nature reserve. This research was funded by the Netherlands Organisation for Scientific Research (NWO-grant 80.33.452 to HdK and NWO-veni-grant 863.08.006 to EJ).

\section{References}

Agrawal AA (2001) Phenotypic plasticity in the interactions and evolution of species. Science 294:321-326 Bakker VJ, Doak DF, Roemer GW, Garcelon DK, Coonan TJ, Morrison SA, Lynch C, Ralls K, Shaw R (2009) Incorporating ecological drivers and uncertainty into a demographic population viability analysis for the island fox. Ecol Monogr 79:77-108 
Blom CWPM, Voesenek LACJ (1996) Flooding: the survival strategies of plants. Trends Ecol Evol 11:290295

Boedeltje G, Bakker JP, ten Brinke A, van Groenendael JM, Soesbergen M (2004) Dispersal phenology of hydrochorous plants in relation to discharge, seed release time and buoyancy of seeds: the flood pulse concept supported. J Ecol 92:786-796

Boyce MS, Haridas CV, Lee CT, NCEAS Stochastic Demography Working Group (2006) Demography in an increasingly variable world. Trends Ecol Evol 21:141-148

Bradshaw AD (2006) Unravelling phenotypic plasticity-why should we bother? New Phytol 170:644-648

Callaway RM, Pennings SC, Richards CL (2003) Phenotypic plasticity and interactions among plants. Ecology 84:1115-1128

Caswell H (1983) Phenotypic plasticity in life-history traits: demographic effects and evolutionary consequences. Am Zool 23:35-46

Caswell H (2001) Matrix population models. Construction, analysis, and interpretation. Sinauer. Sunderland, MA, US

Coulson T, Tuljapurkar S (2008) The dynamics of a quantitative trait in an age-structured population living in a variable environment. Am Nat 172:599-612

Coulson T, Kruuk LEB, Tavecchia G, Pemberton JM, Clutton-Brock TH (2003) Estimating selection on neonatal traits in red deer using elasticity path analysis. Evolution 57:2879-2892

Coulson T, Benton TG, Lundberg P, Dall SRX, Kendall BE, Gaillard JM (2006) Estimating individual contributions to population growth: evolutionary fitness in ecological time. Proc R Soc B 273:547-555

de Kroon H, van Groenendael JM, Ehrlén J (2000) Elasticities: a review of methods and model limitations. Ecology 81:607-618

de Vere N, Jongejans E, Plowman A, Williams E (2009) Population size and habitat quality affect genetic diversity and fitness in the clonal herb Cirsium dissectum. Oecologia 159:59-68

Easterling MR, Ellner SP, Dixon PM (2000) Size-specific sensitivity: applying a new structured population model. Ecology 81:694-708

Ehrlén J (2003) Fitness components versus total demographic effects: evaluating herbivore impacts on a perennial herb. Am Nat 162:796-810

Elderd BD, Doak DF (2006) Comparing the direct and community-mediated effects of disturbance on plant population dynamics: flooding, herbivory and Mimulus guttatus. J Ecol 94:656-669

Ellner SP, Rees M (2006) Integral projection models for species with complex demography. Am Nat 167:410-428

Fox GA, Kendall BE (2002) Demographic stochasticity and the variance reduction effect. Ecology 83:19281934

Franco M, Silvertown J (2004) A comparative demography of plants based upon elasticities of vital rates. Ecology 85:531-538

Horvitz C, Schemske DW, Caswell H (1997) The relative "importance" of life-history stages to population growth: prospective and retrospective analyses. In: Tuljapurkar S, Caswell H (eds) Structured-population models in marine, terrestrial, and freshwater systems. Chapman \& Hall, New York

Huber H, Kane NC, Heschel MS, von Wettberg EJ, Banta J, Leuck AM, Schmitt J (2004) Frequency and microenvironmental pattern of selection on plastic shade-avoidance traits in a natural population of Impatiens capensis. Am Nat 163:548-563

Huber H, Jacobs E, Visser EJW (2009) Variation in flooding-induced morphological traits in natural populations of white clover (Trifolium repens) and their effects on plant performance during soil flooding. Ann Bot 103:377-386

Hutchings MJ, de Kroon H (1994) Foraging in plants: the role of morphological plasticity in resource acquisition. Adv Ecol Res 25:159-238

Jongejans E, de Kroon H (2005) Space versus time variation in the population dynamics of three cooccurring perennial herbs. J Ecol 93:681-692

Jongejans E, de Kroon H, Berendse F (2006) The interplay between shifts in biomass allocation and costs of reproduction in four grassland perennials under simulated successional change. Oecologia 147:369_ 378

Jongejans E, de Vere N, de Kroon H (2008) Demographic vulnerability of the clonal and endangered meadow thistle. Plant Ecol 198:225-240

Knight TM, Barfield M, Holt RD (2008) Evolutionary dynamics as a component of stage-structured matrix models: an example using Trillium grandiflorum. Am Nat 172:375-392

Koons DN, Pavard S, Baudisch A, Metcalf CJE (2009) Is life-history buffering or lability adaptive in stochastic environments? Oikos 118:972-980

Kurashige NS, Callahan HS (2007) Evolution of active and passive forms of plasticity: insights from artificially selected Arabidopsis. Evol Ecol Res 9:935-945 
Lenssen JPM, de Kroon H (2005) Abiotic constraints at the upper boundaries of two Rumex species on a freshwater flooding gradient. J Ecol 93:138-147

Metcalf CJE, Mitchell-Olds T (2009) Life history in a model system: opening the black box with Arabidopsis thaliana. Ecol Lett 12:593-600

Metcalf CJE, Pavard S (2007) Why evolutionary biologists should be demographers. Trends Ecol Evol 22:205-212

Miner BG, Sultan SE, Morgan SG, Padilla DK, Relyea RA (2005) Ecological consequences of phenotypic plasticity. Trends Ecol Evol 20:685-692

Mommer L, Lenssen JPM, Huber H, Visser EJW, de Kroon H (2006) Ecophysiological determinants of plant performance under flooding: a comparative study of seven plant families. J Ecol 94:1117-1129

Morris WF, Doak DF (2004) Buffering of life histories against environmental stochasticity: accounting for a spurious correlation between the variabilities of vital rates and their contributions to fitness. Am Nat $163: 579-590$

Nathan R (2007) Total dispersal kernels and the evaluation of diversity and similarity in complex dispersal systems. In: Dennis AJ, Schupp EW, Green RJ, Westcott DA (eds) Seed dispersal theory and its application in a changing world. CABI, Wallingford

Neitzke M (2001) Analysis of vegetation and nutrient supply in calcareous grassland border zones to determine critical loads for nitrogen. Flora 196:292-303

Neubert MG, Caswell H (2000) Demography and dispersal: calculation and sensitivity analysis of invasion speed for structured populations. Ecology 81:1613-1628

Pelletier F, Clutton-Brock T, Pemberton J, Tuljapurkar S, Coulson T (2007) The evolutionary demography of ecological change: linking trait variation and population growth. Science 315:1571-1574

Pfister CA (1998) Patterns of variance in stage-structured populations: evolutionary predictions and ecological implications. Proc Natl Acad Sci USA 95:213-218

Picotte JJ, Rosenthal DM, Rhode JM, Cruzan MB (2007) Plastic responses to temporal variation in moisture availability: consequences for water use efficiency and plant performance. Oecologia 153:821-832

Pierik R, van Aken JM, Voesenek LACJ (2009) Is elongation-induced leaf emergence beneficial for submerged Rumex species? Ann Bot 103:353-357

Pigliucci M (2001) Phenotypic plasticity: beyond nature and nurture. Johns Hopkins University Press, Baltimore

Pigliucci M, Kolodynska A (2006) Phenotypic integration and response to stress in Arabidopsis thaliana: a path analytical approach. Evol Ecol Res 8:415-433

Rees M, Rose KE (2002) Evolution of flowering strategies in Oenothera glazioviana: an integral projection model approach. Proc R Soc Lond Ser B-Biol Sci 269:1509-1515

Smith M, Caswell H, Mettler-Cherry P (2005) Stochastic flood and precipitation regimes and the population dynamics of a threatened floodplain plant. Ecol Appl 15:1036-1052

Soons MB, Messelink JH, Jongejans E, Heil GW (2005) Habitat fragmentation reduces grassland connectivity for both short-distance and long-distance wind-dispersed forbs. J Ecol 93:1214-1225

Stevens CJ, Dise NB, Mountford JO, Gowing DJ (2004) Impact of nitrogen deposition on the species richness of grasslands. Science 303:1876-1879

Sultan SE (1995) Phenotypic plasticity and plant adaptation. Acta Bot Neerl 44:363-383

Sultan SE (2007) Development in context: the timely emergence of eco-devo. Trends Ecol Evol 22:575-582

Sultan SE, Stearns SC (2005) Environmentally contingent variation: phenotypic plasticity and norms of reaction. In: Hallgrímsson B, Hall BK (eds) Variation: a hierarchical examination of a central concept in biology. Elsevier Academic Press, Amsterdam

Tonsor SJ, Scheiner SM (2007) Plastic trait integration across a $\mathrm{CO}_{2}$ gradient in Arabidopsis thaliana. Am Nat 169:E119-E140

Tuljapurkar S (1990) Population dynamics in variable environments. Springer, New York

Tuljapurkar S, Horvitz CC, Pascarella JB (2003) The many growth rates and elasticities of populations in random environments. Am Nat 162:489-502

van der Sman AJM, Joosten NN, Blom CWPM (1993) Flooding regimes and life-history characteristics of short-lived species in river forelands. J Ecol 81:121-130

van Eck WHJM, van de Steeg HM, Blom CWPM, de Kroon H (2004) Is tolerance to summer flooding correlated with distribution patterns in river floodplains? a comparative study of 20 terrestrial grassland species. Oikos 107:393-405

van Eck WHJM, Lenssen JPM, van de Steeg HM, Blom CWPM, de Kroon H (2006) Seasonal dependent effects of flooding on plant species survival and zonation: a comparative study of 10 terrestrial grassland species. Hydrobiologia 565:59-69

van Kleunen M, Fischer M (2005) Constraints on the evolution of adaptive phenotypic plasticity in plants. New Phytol 166:49-60 
van Kleunen M, Fischer M (2007) Progress in the detection of costs of phenotypic plasticity in plants. New Phytol 176:727-730

van Splunder I, Coops H, Voesenek LAC, Blom CWPM (1995) Establishment of alluvial forest species in floodplains: the role of dispersal timing, germination characteristics and water level fluctuations. Acta Bot Neerl 44:269-278

van Tienderen PH (2000) Elasticities and the link between demographic and evolutionary dynamics. Ecology 81:666-679

Verschoor AM, Vos M, van der Stap I (2004) Inducible defences prevent strong population fluctuations in bi- and tritrophic food chains. Ecol Lett 7:1143-1148

Visser EJW, Bögemann GM, van de Steeg HM, Pierik R, Blom CWPM (2000) Flooding tolerance of Carex species in relation to field distribution and aerenchyma formation. New Phytol 148:93-103

Voesenek LACJ, Rijnders JHGM, Peeters AJM, van de Steeg HMV, de Kroon H (2004) Plant hormones regulate fast shoot elongation under water: from genes to communities. Ecology 85:16-27

Weijschedé J, Martínková J, de Kroon H, Huber H (2006) Shade avoidance in Trifolium repens: costs and benefits of plasticity in petiole length and leaf size. New Phytol 172:655-666 